\title{
Automatic Constructive Appraisal: A Reply to the Commentaries of Parkinson and Kuppens
}

\begin{abstract}
My reply to the comments of Parkinson (2010) and Kuppens (2010) is organized in three parts. The first part deals with Parkinson's claim that the scope of our research is limited because no real emotions were elicited. I suggest that the outcomes in our studies are structurally similar to real emotions but that they lack intensity. In the second part, I try to correct three potential misunderstandings regarding the nature of the comparison process that I proposed. In the third part, I respond to Kuppens' suggestion that we also need to consider the transition of appraisal values into the other components of emotion (actions tendencies, responses, and subjective experience).
\end{abstract}

\section{Keywords}

appraisal, automatic, constructive, emotion

\section{Scope}

Parkinson (2010) questions the scope of our research because our stimuli did not elicit real emotions. According to appraisal theory, stimuli have emotion-eliciting power when they touch on goals/ concerns of a certain importance (e.g., Lazarus, 1991; Moors, 2007). Formally, a stimulus qualifies as an emotion-eliciting stimulus when two elements are present: (a) the stimulus is goal relevant, and (b) the goal is sufficiently important. These elements are rarely present in mainstream research on cognition and emotion. In many studies, stimuli are often words or pictures with a positive or negative valence (e.g., words like "gun" and "birthday" or pictures of a shark or a kitten) and goals are not manipulated. It is therefore impossible to determine whether the stimuli are goal relevant and it is unlikely that they are.

In our studies, goals were manipulated, leading to appraisals of goal congruence/incongruence (or goal-dependent valence). The goal at stake (e.g., winning points, performing well on the task), however, was probably not important enough to create real emotions, at least not intense ones. In other words, the first but not the second element for qualifying stimuli as emotion-eliciting ones was present. I therefore suggest that the stimuli in our studies led to states that are structurally similar to real emotions but lacked intensity.

\section{Nature of the Comparison Process}

I address Parkinson's (2010) criticism about the necessity of constructive/comparison processes to determine goal congruence/ incongruence (or desirability/undesirability, as he calls it) by clarifying three potential misunderstandings. Parkinson writes:

\footnotetext{
Moors argues that priming depends on processes specifically initiated by presentation of the prime, and that the stimulus requires constructive appraisal before any affective significance can be extracted ... participants only appreciate the evaluative significance of a cue indicating success after comparing its representation with a retrieved memory of their current goal.
}

First, I have not argued that priming effects are only produced by processes that occur during or after prime presentation. What I have argued is that conclusions of automaticity can only be drawn for processes that occur during or after prime presentation because only then are processing conditions suboptimal (time is limited, a target response must be prepared).

Second, I have not argued that all affective priming effects are based on constructive processes. In the standard affective priming task, for example, the valence of the primes may be determined by single-input retrieval.

Third, the comparison process that I put forward does not demand that the goal be retrieved from long-term memory. In our experiments, it is more likely that the goal is kept active in short-term memory until the prime is presented. It is even 
possible, as Parkinson (2010) suggests, that goals lead to the prespecification of certain features or (categories of) stimuli as desirable. If so, there are still two possible scenarios. Suppose yellow is prespecified as desirable. According to a fully constructive scenario, any actual feature (yellow or blue color of the prime) is compared to this prespecified feature. According to a semi-constructive scenario, when the actual feature is yellow, it can be recognized as desirable without the need for a comparison process; when it is blue, it must be compared with the prespecified feature to determine that it is undesirable (because it was not prespecified as undesirable). There is of course a third scenario, which is entirely nonconstructive. According to this scenario, one feature (e.g., yellow) is prespecified as desirable and the other (e.g., blue) as undesirable. Here, the recognition of actual features as desirable or undesirable does not require a comparison process. Which of these three scenarios is correct is an empirical question that we addressed (partly) in the studies reported in Moors, De Houwer, and Eelen (2004). These studies provide more support for a constructive (and/or semi-constructive) explanation than for a nonconstructive one, but they do not rule out every possible alternative scenario that might fall under the label of nonconstructive. Leaving aside questions about the persuasiveness of our findings, the main goal was to put the issue of the automaticity of constructive appraisal on the agenda. Future studies may or may not confirm the conclusions drawn so far.

I further wish to note that the comparison process put forward need not be a one-time shot. It may well be recurrent. To still qualify as a comparison process, however, a comparison must take place during each cycle (cf. TOTE model; Miller, Galanter, \& Pribram, 1960). During the act of pushing against a jammed door, subtle changes may occur in the actual state (as the resistance of the door may fluctuate) and perhaps even in the desired state (at one point, the desired state may be that the door opens; at another, it may be that the door does not open too abruptly). In my view, the fact that in many real-life situations actual states and desired ones vary quickly over time, argues in favour of, rather than against, the plausibility of a fast comparison process. If we would need to store and label all possible combinations of (un)desired and actual states, our storage capacity would be heavily charged.

On a final note, the goal entering in the stimulus-goal comparison need not be represented in a symbolic (verbal-like) format and it need not be conscious. To qualify as a goal representation, however, it has to have dynamic qualities that are not shared by other kinds of representations (e.g., the activation of goal representations accumulates over time and persists in the face of obstacles; Bargh \& Barndollar, 1996).

\section{Two Steps in Emotion Causation}

I agree with Kuppens (2010) that theories of emotion causation should ideally account for two steps within the emotion causation process: the evaluation of the emotion-eliciting event and the transition from the evaluation into the other components of emotions (action tendencies, responses, subjective experience; cf. Moors, 2009). I also agree that our research only addresses the first step. From the viewpoint of appraisal theory, this is the most important step. As Kuppens correctly notes, appraisal theorists often assume that the transition from the first to the second step is fairly seamless. Action tendencies follow directly from the outcomes of appraisal components and are materialized in physiological and expressive responses. Subjective experience is pictured as a simple read-out of the other components (appraisal, action tendencies, and responses). The validity of this picture remains open to empirical testing.

\section{References}

Bargh, J. A., \& Barndollar, K. (1996). Automaticity in action: The unconscious as repository of chronic goals and motives. In P. M. Gollwitzer \& J. A. Bargh (Eds.), The psychology of action: Linking cognition and motivation to behavior (pp. 457-481). New York: Guilford Press.

Kuppens, P. (2010). From appraisal to emotion. Emotion Review, 2(2), $157-158$.

Lazarus, R. S. (1991). Emotion and adaptation. New York: Oxford University Press.

Miller, G. A., Galanter, E., \& Pribram, K. A. (1960). Plans and the structure of behavior. New York: Holt, Rhinehart, \& Winston.

Moors, A. (2007). Can cognitive methods be used to study the unique aspect of emotion: An appraisal theorist's answer. Cognition \& Emotion, 21, 1,238-1,269.

Moors, A. (2009). Theories of emotion causation: A review. Cognition \& Emotion, 23, 625-662.

Moors, A., De Houwer, J., \& Eelen, P. (2004). Automatic stimulus-goal comparisons: Support from motivational affective priming studies. Cognition \& Emotion, 18, 29-54.

Parkinson, B. (2010). Recognizing desirability: Is goal comparison necessary? Emotion Review, 2(2), 159-160. 